\title{
Prevalencia de demencia en una población urbana de Lima-Perú: estudio puerta a puerta
}

\author{
Dementia prevalence in a Lima, Peru urban community: door-to-door study
}

\author{
Nilton Custodio ${ }^{1,2}$, Alberto García ${ }^{3}$, Rosa Montesinos ${ }^{1}$, Juan Escobar ${ }^{1,4}$, Liliana Bendezú ${ }^{2}$ \\ ${ }^{1}$ Unidad de Diagnóstico de Deterioro Cognitivo y Prevención de Demencia, Clínica Internacional, Lima, Perú. \\ ${ }^{2}$ Servicio de Neurología, Clínica Internacional, Lima, Perú. \\ ${ }^{3}$ Servicio de Neurología, Instituto Médico Miraflores, Lima, Perú. \\ ${ }^{4}$ Facultad de Medicina, Universidad Nacional Mayor de San Marcos, Lima, Perú
}

\begin{abstract}
Resumen
Objetivos: Calcular la prevalencia y tipos de demencia en una población de una comunidad urbana de Lima. Diseño: Muestreo por conglomerados bietápico. Instituciones: Instituciones de salud privadas. Pacientes: Individuos mayores de 65 años. Intervenciones: En el distrito de Cercado del Lima, 1532 individuos mayores de 65 años, seleccionados aleatoriamente, fueron sometidos a evaluaciones neuropsicológicas breves, que incluyeron el Mini Mental State Examination (MMSE), la prueba del dibujo del reloj (PDR) y el cuestionario de actividades funcionales de Pfeffer (PFAQ). Según los resultados obtenidos luego de la aplicación del MMSE, PDR y PFAQ, los individuos fueron clasificados como seleccionados y no seleccionados, siendo el grupo de seleccionados aquellos que presentaban deterioro cognitivo luego de la aplicación de dichas pruebas. El grupo de personas seleccionadas fueron sometidas a evaluaciones neurológicas, neuropsicológicas, exámenes de laboratorio y tomografía computada (TC) cerebral. Principales medidas de resultados: Prevalencia de demencia. Resultados: Encontramos 105 casos de demencia, lo que corresponde a una prevalencia de 6,85\%, siendo la enfermedad de Alzheimer el diagnóstico más frecuente $(56,2 \%)$; dicha prevalencia se incrementó con la edad y tuvo predominio en el sexo femenino. El análisis de regresión logística mostró que la edad, género femenino y nivel educativo bajo se encontraban correlacionados con la prevalencia alta de demencia. Conclusiones: La prevalencia de demencia en esta población peruana fue 6,85\% y la enfermedad de Alzheimer, el diagnóstico más frecuente; la edad, el género femenino y el nivel educativo bajo fueron los factores correlacionados con esta prevalencia alta.
\end{abstract}

Palabras clave: Demencia; enfermedad de Alzheimer; epidemiologia; educación.

\begin{abstract}
Objective: To determine the prevalence and types of dementia in a Lima's urban community. Design: Bietapic conglomerate sampling. Setting: Private health institutions. Patients: Older than 65 years subjects. Interventions: In the Cercado de Lima district, 1532 older than 65 years subjects randomly selected had brief neuropsychological evaluations including the Mini Mental State Examination (MMSE), drawing the Clock Test (PDR), and Pfeffer's functional activities questionnaire (PFAQ). According to the results obtained, individuals were classified as selected or not selected. The group selected included those subjects presenting/displaying cognitive deterioration, who further had neurological and neuropsychological evaluations, laboratory tests and brain computed tomography (TC). Main outcome measures: Dementia prevalence. Results: We found 105 cases of dementia corresponding to $6,95 \%$ prevalence; Alzheimer's disease was the most frequent diagnosis $(56,2 \%)$. Dementia prevalence increased with age mainly in females. Logistic regression analysis showed that age, female sex and low education correlated with dementia prevalence. Conclusions: Dementia prevalence in this Peruvian population was $6,85 \%$, and Alzheimer's disease the most frequent diagnosis; age, female gender and low educative level factors correlated with this high prevalence.
\end{abstract}

Key words: Dementia; Alzheimer disease; epidemiology; education.

\section{INTRODUCCIÓN}

Teniendo en cuenta que la expectativa de vida de la población mundial es cada vez mayor, es necesario conocer el impacto en los sistemas de salud de las enfermedades degenerativas de la población de la tercera edad, y la demencia es uno de los principales problemas. En países desarrollados, la prevalencia cruda de demencia en pobladores mayores de 65 años de edad es de $6 \%$ a $9 \%{ }^{(1,2)}$; siendo más baja en China, África e India ${ }^{(3-5)}$.

En América Latina, los estudios basados en población son escasos; sin embargo, han servido de base para establecer la prevalencia de demencia, la cual fluctúa entre $4,03 \%$ y $8,2 \%$. Ketzoian y colaboradores, en 2731 individuos mayores de 65 años de edad encontraron una prevalencia de 4,03\%, en la población de Villa del Cerro, Montevideo, Uruguay ${ }^{(6)}$. En Cuba, Llibre y colaboradores, en 1139 individuos de la misma edad hallaron una prevalencia de demencia de 8,2\%, en la población de La Habana ${ }^{(7)}$. Herrera y colaboradores, en Catanduva, Sao Paulo, Brasil, en 1656 individuos mayores de 65 años de edad encontraron una prevalencia de demencia de $7,1 \%{ }^{(8)}$. En el Perú, no existen estudios basados en población.

Los objetivos de la presenta investigación son determinar la prevalencia de demencia y la frecuencia relativa de los tipos de demencia en una población urbana del Cercado de Lima, Perú, y luego establecer su correlación con edad, género y años de estudio.

\section{MÉTODOS}

Se realizó un estudio observacional, transversal en el Cercado de Lima donde, según el censo de 2005, vive una población de 289855 habitantes, de los cuales 29165 tienen igual o más de 65 años de edad. Teniendo en cuenta una prevalencia esperada de $7 \%$ en adultos mayores de 65 años de edad, 2958 individuos deberían ser evaluados.

Se realizó un muestreo bietápico por conglomerados. La unidad primaria de muestreo fueron las manzanas; la unidad secundaria de muestreo fueron las viviendas; las unidades de observación fueron las personas mayores de 65 años que cumplieron los criterios de inclusión de la fase I y las unidades informantes 
fueron las personas seleccionadas y los habitantes de la vivienda o los cuidadores habituales (en caso de ser requerido). Para determinar el número de unidades primarias de muestreo a seleccionar, se estableció que por cada manzana se seleccionara 4 viviendas de manera aleatoria. Un supuesto que planteamos es que en cada vivienda habitan en promedio 2 personas mayores de 65 años. Entonces, el número de unidades secundarias de muestreo a ser seleccionadas fueron 1479 . Así, determinamos que debíamos seleccionar 370 manzanas, distribuidas proporcionalmente al número de manzanas con que cuenta cada distrito de la jurisdicción de la dirección de Salud, Lima-Ciudad. La participación de la población fue voluntaria, y todos ellos fueron visitados previamente para obtener un consentimiento informado directamente del individuo evaluado o de un miembro de la familia o del cuidador habitual.

Un total de 43 entrevistadores, entre estudiantes de medicina de la Universidad Nacional Mayor de San Marcos y Universidad Peruana Cayetano Heredia (26), médicos generales recién graduados (11) y residentes de la especialidad de Geriatría de la Universidad Nacional Mayor de San Marcos (6) fueron entrenados apropiadamente por el investigador principal para realizar las pruebas de cribado. Los entrevistadores recibieron entrenamiento en Mini-mental State Examination (MMSE)

(9), Prueba de Dibujo del Reloj-versión de Manos (PDR-M) ${ }^{(10)}$ y Pfeffer Functional Activities Questionnaire (PFAQ) (11), durante una semana antes de realizar las pruebas en el campo, después de lo cual fueron evaluados para establecer la confianza inter-evaluador, el cual fue establecido como superior a 0,90 .

La fase I de la evaluación incluyó un cuestionario sobre datos demográficos y socio-económicos y luego información sobre el estado mental del individuo evaluado, otorgado por él mismo, por un miembro de la familia o por el cuidador habitual. La evaluación del estado mental incluyó MMSE, PDR-M y PFAQ. El punto de corte en el MMSE para sospecha de demencia fue ajustado según años de educación: 27 para individuos con más de 7 años de educación, 23 para aquellos con 4 a 7 años de educación, 21 para aquellos con 1 a 3 años de educación y 18 para los iletrados. La PDR-M evalúa la capacidad del individuo para poner sobre un círculo dibujado los números del 1 al 12, tal como van en un reloj, y luego evalúa la dirección y proporcionalidad de las manecillas del reloj al intentar plasmar las 11:10 horas. El puntaje máximo es 10 y un puntaje menor a 8 indica compromiso cognitivo. El PFAQ incluye 10 preguntas acerca de las actividades de la vida diaria, con rangos de puntaje de 0 a 3, según la severidad de la discapacidad en cada actividad. El puntaje máximo es 30 y un puntaje mayor de 5 indica compromiso funcional.

Los sujetos que presentaron puntajes compatibles con sospecha de demencia en por lo menos una de las 3 pruebas de cribado fueron seleccionados para la fase II del estudio. Ellos fueron evaluados por los neurólogos autores del presente estudio, quienes realizaron la historia clínica, el examen físico general y neurológico, con particular énfasis en el aspecto cognitivo. Se realizó un segundo MMSE, una segunda PDR-M y la siguiente batería neuropsicológica: Rey Auditory Verbal Learning Test, Logical Memory Subtest (Wechsler Memory Scale revised), Trail Making Test A y B, Figura Compleja de Rey, Test de Denominación de Boston, Wisconsin Card Sorting Test, Letter-Number (Subtest Weschler Adult Intelligent Scale III), Digit Span. La evaluación también incluyó la escala isquémica de Hachisnki ${ }^{(12)}$ y el Clinical Dementia Rating Scale (CDR) ${ }^{(13)}$, para evaluar la severidad de la demencia. En esta fase, se excluyó los pacientes con dificultad para realizar las pruebas cognitivas, debido a problemas auditivos, visuales u otros problemas físicos que pudieran interferir con su performance y aquellos con lengua diferente al español. Los datos obtenidos en la fase II fueron analizados por los investigadores del estudio, y los sujetos que completaron los criterios del Diagnostic and Statistical Manual of Mental Disorders (volumen IV; DSM IV) fueron seleccionados para la fase III.

En la fase III, los pacientes con demencia fueron sometidos a un plan de trabajo para descartar pseudodemencia o demencia tratable. Los exámenes sanguíneos incluyeron hemograma, hemoglobina, hematocrito, glucosa, urea, creatinina, perfil hepático, perfil lipídico, hormonas tiroideas, niveles séricos de vitamina B12 y ácido fólico, serología para sífilis, ElisaVIH y tomografía computarizada cerebral sin contraste.

Basados en los resultados de la historia clínica, el examen neurológico, los resultados de exámenes sanguíneos y la tomografía computarizada cerebral se aplicaron los criterios previamente publicados del National Institute of Neurological Communicative Disorders and StrokeAlzheimer's Disease and Related Disorders Association (NINCDS-ADRDA) ${ }^{(14)}$ para Enfermedad de Alzheimer (EA), del National Institute of Neurological Disorders and Stroke-Association Internationale pour la Recherche et L'Enseignement en Neurosciences (NINDS-AIREN) ${ }^{(15)}$ para demencia vascular (DV) y para EA con enfermedad cerebro-vascular (EA+ECV), de McKeith ${ }^{(16)}$ del Consorcio para el diagnóstico de Demencia con Cuerpos de Lewy (DCL), y de Neary ${ }^{(17)}$ del Consenso para Demencia Fronto-Temporal (DFT). En los casos de pacientes con EA, se evaluó además el aspecto cognitivo, según ADAS-cog ${ }^{(18)}$, y los síntomas conductuales con NPI ${ }^{(19)}$.

Para el análisis estadístico, en el caso de variables cuantitativas, se determinó primero la normalidad de la distribución, mediante la prueba de KolmogorovSmirnov. Cuando resultó una distribución normal, se aplicó la prueba t de student; $y$, en caso de una distribución no normal, se empleó la prueba U de MannWhitney. Para las variables cualitativas, se empleó la prueba de chi cuadrado. Las variables que obtuvieron un $p<0,01$ fueron incorporadas a un modelo de regresión logística condicional, mediante el cual se obtuvo el $O R$ ajustado para cada factor y su intervalo de confianza al 95\%. Se utilizó el programa informático SPSS 12,0.

\section{RESULTADOS}

Un total de 1839 sujetos aceptaron ser evaluados; sin embargo, 123 retiraron su consentimiento antes de iniciar la evaluación, 83 sufrían deficiencia visual severa, 52 deficiencia auditiva severa y 49 información inadecuada; por lo que, 1532 sujetos fueron evaluados en la fase I. 
Tabla 1. Distribución por edad y género de 1532 sujetos evaluados en la fase 1.

\begin{tabular}{cccc}
\hline Grupo de edad (años) & $\mathrm{n}(\%)$ & Femenino (\%) & Masculino (\%) \\
\hline 65 a 69 & $582(37,9)$ & $341(58,6)$ & $241(41,4)$ \\
70 a 74 & $428(27,9)$ & $243(56,8)$ & $185(43,2)$ \\
75 a 79 & $252(16,5)$ & $148(58,7)$ & $104(41,3)$ \\
80 a 84 & $179(11,7)$ & $110(61,5)$ & $69(38,5)$ \\
85 a 96 & $91(5,9)$ & $54(59,3)$ & $37(40,7)$ \\
Total & $1532(100,0)$ & $896(58,5)$ & $636(41,5)$ \\
\hline
\end{tabular}

Tabla 2. Distribución por años de educación y género de 1532 sujetos evaluados en la fase 1.

\begin{tabular}{cccc}
\hline Años de educación & $\mathrm{n}(\%)$ & Femenino $(\%)$ & Masculino $(\%)$ \\
\hline lletrados & $269(17,5)$ & $181(67,3)$ & $88(32,7)$ \\
1 a 3 & $312(20,4)$ & $213(68,3)$ & $99(31,7)$ \\
4 a 7 & $417(27,2)$ & $225(53,9)$ & $192(46,1)$ \\
Más de 8 & $534(34,9)$ & $277(51,9)$ & $257(48,1)$ \\
Total & $1532(100,0)$ & $896(58,5)$ & $636(41,5)$ \\
\hline
\end{tabular}

Como se puede observar en la tabla 1 , casi 38\% de los 1532 sujetos tenía entre 65 y 69 años de edad, mientras que solo $5,9 \%$ tenía entre 85 y 96 años. Respecto a género, $58,5 \%$ de la muestra era de sexo femenino, siendo esta mayor $(61,5 \%)$ en el grupo de edades entre 80 y 84 años. La evaluación de los 1532 sujetos según años de educación reveló que $269(17,5 \%)$ eran iletrados, $312(20,4 \%)$ tuvieron entre 1 a 3 años de educación, 417 $(27,2 \%)$ entre 4 a 7 años de educación y 534 (34,9\%) habían tenido más de 8 años de educación (tabla 2). La proporción de iletrados fue más frecuente en el sexo femenino $(67,3 \%)$.

Considerando MMSE, PDR-M y PFAQ; 229 pacientes fueron seleccionados para la fase II, correspondiendo a $14,9 \%$ de los sujetos evaluados en la fase I. De los 229 pacientes seleccionados de la fase I, 212 (92,6\%) completaron la fase II. Ciento siete pacientes fueron descartados por ser considerados normales o sin evidencia de demencia, incluyendo 12 casos de depresión y 3 casos de rendimiento cognitivo bajo por uso de biperideno, los cuales según la evolución del cuadro clínico tras el tratamiento apropiado y el medicamento retirado en cada caso, fueron catalogados como pseudodemencia. De los 105 sujetos considerados para la fase 3, dos descontinuaron el estudio (uno por retiro de consentimiento y el otro por cambio de dirección).

Luego de la evaluación consensual, 103 casos fueron diagnosticados como demencia. Doce de estos pacientes presentaron enfermedades concomitantes: depresión en 9 casos, deficiencia de vitamina B12 en 2 y un caso de hipotiroidismo. Sin embargo, el tratamiento apropiado no produjo cambios en el rendimiento cognitivo.

Teniendo en cuenta que fueron evaluados 1532 sujetos y 105 los casos diagnosticados como demencia, encontramos

Tabla 3. Causas de demencia en

\begin{tabular}{lrr}
\hline Diagnóstico & $\mathrm{n}$ & $\%$ \\
\hline EA probable & 51 & 49,5 \\
EA posible & 7 & 6,8 \\
Demencia vascular & 9 & 8,7 \\
EA con ECV & 16 & 15,5 \\
DEP & 3 & 2,9 \\
DCL & 2 & 1,9 \\
DFT & 2 & 1,9 \\
No determinado & 13 & 12,7 \\
\hline
\end{tabular}

$E A=$ Enfermedad de Alzheimer $E C V=$ Enfermedad cerebro-vascular. $D E P=$ Demencia por enfermedad de Parkinson. $D C L=$ Demencia con cuerpos de Lewy. $D F T=$ Demencia fronto-temporal. 103 casos diagnosticados. una prevalencia de 6,85\%. Al evaluar las causas de demencia, 58 casos correspondieron a EA, lo cual representa 56,3\% (51 casos con EA probable y 7 casos con EA posible); 9 casos correspondieron a DV y 16 casos, a EA con ECV (8,7\% y $15,5 \%$, respectivamente) (tabla 3 ).

Según la escala CDR, 41 (39,8\%) pacientes fueron clasificados como demencia leve (CDR 1), 36 (34,9\%) como moderada (CDR 2), y 26 (25,3\%) como severa (CDR 3). En 37 casos de los 58 casos de EA, se realizó ADAS-cog. Encontramos 6 casos $(16,2 \%)$ con EA leve (ADAS-cog 16-20), 18 casos $(48,6 \%)$ con EA moderada (ADAS-cog 21-30), 8 casos $(21,6 \%)$ con EA moderadamente severa (ADAS-cog 31-45) y 5 casos $(13,5 \%)$ con EA severa (ADAS-cog > 45) (tabla 4).

Tabla 4. Severidad de demencia en 37 casos de Enfermedad de Alzheimer según el aspecto cognitivo evaluado por ADAS-cog ${ }^{(18)}$.

\begin{tabular}{lcc}
\hline Severidad & $\mathrm{n}$ & $\%$ \\
\hline ADAS-cog 16 a 20 & 6 & 16,2 \\
ADAS-cog 21 a 30 & 18 & 48,6 \\
ADAS-cog 31 a 45 & 8 & 21,6 \\
ADAS cog > 45 & 5 & 13,5 \\
\hline
\end{tabular}

En nuestra serie de pacientes, la prevalencia de demencia se asoció al envejecimiento (tabla 5). De la misma manera, la demencia fue más prevalente entre mujeres y en individuos iletrados (tabla 6). El análisis de regresión logística demostró que la edad $(p=0,000)$, género femenino $(p=0,030)$ y niveles bajos de educación $(p=0,003)$ se asociaron con prevalencia alta de demencia.

\section{DISCUSIÓN}

En el presente estudio, la prevalencia de demencia en una comunidad urbana de la ciudad de Lima fue $6,85 \%$, similar a la encontrada en estudios realizados en América Latina ${ }^{(6-8)}$. Estudios epidemiológicos previos de prevalencia de demencia en individuos mayores de 65 años de edad varían entre 2,2\% en África, 5,5\% en Asia, $6,4 \%$ en América del Norte, $7,1 \%$ en 
Tabla 5. Prevalencia de demencia en relación a edad.

\begin{tabular}{cccc}
\hline $\begin{array}{c}\text { Grupo de edad } \\
\text { (años) }\end{array}$ & $\mathrm{n}$ & $\begin{array}{c}\text { Demencia } \\
(\mathrm{n})\end{array}$ & $\begin{array}{c}\text { Demencia } \\
\%(95 \% \mathrm{Cl})\end{array}$ \\
\hline 65 a 69 & 582 & 6 & $1,1(1,0-1,3)$ \\
70 a 74 & 428 & 9 & $2,1(1,9-2,4)$ \\
75 a 79 & 252 & 21 & $8,3(7,9-8,5)$ \\
80 a 84 & 179 & 26 & $14,5(14,2-14,9)$ \\
Más de 85 & 91 & 41 & $45,1(44,6-45,8)$ \\
\hline$X^{2}=221,17 ; p=0,0000$ & & &
\end{tabular}

América del Sur y 9,4\% en Europa ${ }^{(20)}$. Las comunicaciones de prevalencia varían entre las diversas regiones, e inclusive entre países de una misma región, y peor aún dentro de un mismo país. Pero, lo que sí está claro es que existe prevalencia alta en países de Europa ${ }^{(3)}$ y baja en países de África ${ }^{(3,4)}$ y Asia ${ }^{(4)}$. En Europa, se comunica en Holanda 6,5\% ${ }^{(21)}$, Dinamarca $7,1 \%{ }^{(22)}$, Bélgica $9 \%{ }^{(23)}$ y en España $5,5 \%{ }^{(24)}, 13,9 \%{ }^{(25)}$ y $14,9 \%{ }^{(26)}$. En China, la prevalencia en individuos mayores de 65 años de edad fue 3,5\% para EA y $1,1 \%$ para DV ${ }^{(4)}$. En los estudios latinoamericanos, en Uruguay, Ketzoian y colaboradores ${ }^{(6)}$, en Cuba, Llibre y colaboradores ${ }^{(7)}$, y en Brasil, Herrera y colaboradores ${ }^{(8)}$ demostraron que la prevalencia en individuos mayores de 65 años de edad fue $4,03 \%, 7,1 \%$ y $8,2 \%$, respectivamente. Las variaciones en las tasas de prevalencia pueden tener su origen en los diferentes criterios diagnósticos usados en la recolección de datos, en las diferencias culturales y regionales o étni- cas. Así, en los estudios de Ketzoian ${ }^{(6)}$, el diagnóstico de demencia fue basado en MMSE, tests neuropsicológicos y en la opinión de expertos; en los estudios de Llibre ${ }^{(7)}$, el diagnóstico de demencia se basó en los criterios del DSM-III revisado; mientras que en los estudios de Herrera ${ }^{(8)}$, se basó en los criterios del DSM-IV. En nuestra investigación, usamos los criterios del DSM IV para demencia y los criterios del NINCDSADRDA para EA, criterios similares a los usados por el grupo de Herrera. Sin embargo, encontramos diferencias en la proporción de individuos iletrados, pues en nuestra investigación estos representaron $17,5 \%$, mientras que en el grupo de pacientes de Catanduva alcanzaron $34,2 \%$. Estas diferencias podrían ser explicadas por la marcada tendencia al centralismo de las políticas educativas en el Perú. Además, con la finalidad de aumentar la probabilidad de detectar individuos con sospecha de demencia, nosotros agregamos la PDR-M durante la fase I.

Tabla 6. Prevalencia de demencia en relación a género y años de educación.

\begin{tabular}{lccc}
\hline & $n$ & $\begin{array}{c}\text { Demencia } \\
(\mathrm{n})\end{array}$ & $\begin{array}{c}\text { Demencia } \\
(\%)\end{array}$ \\
\hline Género & & & \\
Masculino & 636 & 29 & 4,6 \\
Femenino & 896 & 74 & 8,3 \\
$X^{2}=5,8 ; p=0,001$ & & & \\
& & & \\
Años de educación & & & \\
lletrados & 269 & 41 & 15,2 \\
1 a 3 años & 312 & 25 & 8,1 \\
4 a 7 años & 417 & 17 & 3,1 \\
Más de 8 años & 534 & 20 & \\
$X^{2}=32,37 ; p=0,0000$ & & & \\
\hline
\end{tabular}

La enfermedad de Alzheimer fue la causa más frecuente de demencia en nuestra serie de pacientes, representando $56,3 \%$ de los casos, seguido por EA+ECV $(15,5 \%)$ y DV $(8,7 \%)$. Nuestros resultados son similares a los previamente hallados a nivel mundial $(2,3,27)$. No obstante, el hecho de no haber realizado imágenes por resonancia magnética de cerebro puede distorsionar la verdadera proporción de individuos con $\mathrm{EA}+\mathrm{ECV}$ y DV. Sin embargo, de manera similar en el estudio de Herrera ${ }^{(8)}$ y colaboradores la proporción de pacientes con EA respecto de EA+ECV y DV llega a ser 3 veces mayor, lo cual puede tener 2 posibles explicaciones: a) que realmente nuestra región presente esta proporción; o b) que nuestros estudios incluyan pacientes de edad avanzada. Son necesarios estudios adicionales en América del Sur para aclarar estos supuestos. Por otro lado, los estudios asiáticos han revelado una inversión en la proporción de pacientes con demencia. En el estudio en Hisayama, la tasa de pacientes con DV comparado con EA varían de 1,8:1 a 1,1:1 durante los años del estudio, 85 al $92^{(28)}$. Sin embargo, un reciente estudio en China encuentra una prevalencia de $4,8 \%$ para EA y $1,1 \%$ para DV ${ }^{(4)}$. Estos resultados son comparables a las tasas estandarizadas para edad de 1,6\% para DV, en 11 estudios europeos realizados por más de 10 años ${ }^{(27)}$.

El 61,2\% de la población con demencia tenía una enfermedad moderada a severa, mientras que en los pacientes con EA, el 83,8\% presentó estadios moderados a severos. Estos resultados coinciden con investigaciones publicadas, en las cuales el sub-diagnóstico es frecuente, tanto por los encargados de los servicios primarios de salud ${ }^{(29)}$ como por la falta de reconocimiento de los síntomas por los familiares ${ }^{(30)}$, quienes acuden a la consulta cuando hacen su aparición los síntomas conductuales. En una investigación, $78,6 \%$ de individuos con demencia leve, $71,4 \%$ con demencia moderada y $20 \%$ con demencia severa no tenían documentado un diagnóstico de demencia $^{(31)}$.

La prevalencia de demencia se incrementa con la edad, de 1,1\% (95\% CI: 1,0-1,3) en el grupo etario de 65 a 
69 años de edad hasta 45,1\% (95\% CI: 44,6-45,8)en aquellos de más de 85 años de edad. Estos resultados confirman los estudios previamente publicados, que sugieren que las tasas de prevalencia de demencia aumentan exponencialmente con el aumento de la edad ${ }^{(8,32,33)}$. El análisis de género confirma la tendencia anteriormente descrita ${ }^{(8,32)}$, en la cual la prevalencia de demencia fue significativamente más alta en mujeres. Se ha concluido ${ }^{(34)}$ que el mayor número de mujeres con EA podría ser debido a la mayor expectativa de vida de las mujeres. Sin embargo, son necesarios más estudios al respecto para aclarar este tema.

Respecto a la relación de demencia con años de estudio, nuestra muestra mostró una prevalencia de demencia de 3,7\% en individuos con más de 8 años de educación, mientras que alcanzó 15,2\% entre los iletrados. De esta manera, el analfabetismo se asoció con una significativa prevalencia alta de demencia, asociación independiente cuando se realizó el análisis multivariado con edad y género femenino. Si bien es cierto que estos hallazgos podrían reflejar un sobre-diagnóstico de demencia entre personas con menor nivel de educación, quienes tendrían menor capacidad para realizar adecuadamente las evaluaciones neuro-psicológicas, nosotros basamos el diagnóstico de demencia, no solo en el resultado de dichas evaluaciones, pues además fundamentamos el diagnóstico de demencia en la menor capacidad de los individuos para realizar las actividades de la vida diaria; lo cual hace poco probable esta presunción. Muchos son los estudios que han demostrado estrecha asociación entre niveles bajos de educación y demencia ${ }^{(2-4,8,35,36)}$. Sin embargo, existen algunos estudios que han fallado en demostrar esta asocia-

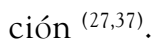

En una reciente investigación (38), los factores de confusión relacionados a características socio-económicas, vasculares o estilos de vida entre personas con diferentes niveles de educación no han influenciado en el desarrollo de demencia, lo cual sugiere que el efecto de la educación es independiente de otros factores de riesgo para demencia. Se plantea que personas con elevados niveles de educación tienen gran reserva cognitiva, lo cual puede posponer las manifestaciones clínicas de demencia.

En conclusión, la prevalencia de demencia en la población peruana del Cercado de Lima es similar a los estudios previamente publicados, y la enfermedad de Alzheimer fue el diagnóstico más frecuente. La edad, género femenino y nivel bajo de educación se asociaron con la prevalencia de demencia.

\section{AGRADECIMIENTO}

Los autores agradecen al Profesor Ricardo Nitrini por su asesoría en el diseño de la investigación, a los alumnos de la facultad de Medicina Humana de la Universidad Nacional Mayor de San Marcos y de la Universidad Peruana Cayetano Heredia por su participación en la fase 1 del estudio, y al Dr. César Gutiérrez por las sugerencias en el análisis estadístico.

\section{REFERENCIAS BIBLIOGRÁFICAS}

1. Kawas $\mathrm{CH}$, Katzman R. Epidemiology of dementia and Alzheimer's disease. En: Terry RD, Katzman R, Bick KL, Sisodia SS, eds. Alzheimer's disease. 2nd ed. Philadelphia: Lippincot Williams Wilkins, 1999. p. 95-116.

2. Berr C, Wancata J, Ritchie K. Prevalence of dementia in the elderly in Europe. Eur Neuropsychopharmacol. 2005;15:463-71.

3. Ferri CP, Prince M, Brayne C, Brodaty H, Fratiglioni L, Ganguli M, et al. Global prevalence of dementia: a Delphi consensus study. Lancet. 2005;366:21127.

4. Zhang ZX, Zahner GE, Roman GC, Liu J, Hong Z, Qu $Q M$, et al. Dementia subtypes in China: prevalence in Beijing, Xian, Shangai and Chengdu. Arch Neurol. 2005;62:447-53.

5. Shaji S, Bose S, Verghese A. Prevalence of dementia in an urban population in Kerala, India. Br J Psychiatry. 2005;186:136-40.

6. Ketzoian C, Rega I, Caseres R. Estudio de prevalencia de las principales enfermedades neurológicas en una población del Uruguay. La Prensa Médica Uruguaya. 1997;17:9-26.

7. Llibre JJ, Guerra MA, Perez-Cruz H, Bayarre $\mathrm{H}$, Fernandez S, Gonzales M, et al. Dementia syndrome and risk factors in adults older than 60 years old residing in Habana. Rev Neurol. 1999;29:908-11.

8. Herrera E, Caramelli P, Barreiros AS, Nitrini R. Epidemiologic survey of dementia in a communitydwelling Brazilian population. Alzheimer Dis Assoc Disord. 2002;16:103-8.
9. Folstein MF, Folstein SE, McHugh PR. "Mini-mental state": a practical method for grading the cognitive state of patients for the clinician. J Psychiat Res. 1975;12:189-98.

10. Manos P, Wu R. The ten-point clock test: A quick screen and grading method for cognitive impairment in medical and surgical patients. Int J Psych Med. 1994;24:229-44.

11.Pfeffer RI, Kurosaki TT, Harrah CH Jr, Chance JM, Filos S. Measurement of functional activities in older adults in the community. J Gerontol. 1982;37:323-9.

12. Hachinski VC, lliff LD, Zilhka E, Do Boulay GHD, MC Allister, Marshall J, et al. Cerebral blood flow in dementia. Arch Neurol. 1975;32:632-7.

13. Hughes CP, Berg L, Danziger WL, Coben LA, Martin $\mathrm{RL}$. A new clinical scale for the staging of dementia. Br J Psychiatr. 1982;140:566-72.

14. McKhann G, Drachman D, Folstein M, Katzman R, Price D, Stadlan EM. Clinical diagnosis of Alzheimer's disease: report of the NINCDS-ADRDA work group under the auspices of department of health and human services task force on Alzheimer's disease. Neurology. 1984;34:939-44.

15. Roman GC, Tatemichi TK, Erkinjuntti T, Cummings JL, Masdeu JC, Garcia JH. Vascular dementia: diagnostic criteria for research studies. Report of the NINDS-AIREN international workshop. Neurology. 1993;43:250-60.

16. McKeith IG, Galasko D, Kosaka K, Perry EK, Dickson DW, Hansen LA, et al. Review. Consensus guidelines for the clinical pathologic diagnosis of dementia with Lewy bodies (DLB): report of the consortium on (DLB) international workshop. Neurology. 1996;47:1113-24.

17. Neary D, Snowden JS, Gustafson L, Passant U, Stuss D, Black S, et al. Frontotemporal lobar degeneration: a consensus on clinical diagnostic criteria. Neurology. 1998;51:1546-54.

18. Rosen WG, Mohs RC, Davis KL. A new rating scale for Alzheimer's disease. Am J Psychiatry. 1984;141:1356-64.

19. Cummings JL, Mega M, Gray K, RosenbergThompson S, Carusi DA, Gornbein J. The neuropsychiatric inventory: comprehensive assessment of psychopathology in dementia. Neurology. 1994;44:2308-14.

20. Lopes MA, Bottino CMC. Prevalencia de demencia em diversas regiones do mundo: Analise dos estudos epidemiológicos de 1994 a 2000. Arq Neuropsiquiatr. 2002;60:61-9.

21. Boersma F, Eefsting JA, van den Brink W, Koeter M, van Tilburg W. Prevalence of dementia in a rural Netherlands population and the influence of DSMIII-R and CAMDEX criteria for the prevalence of mild and more severe forms. J Clin Epidemiology. 1998;51:189-97.

22. Andersen K, Lolk A, Nielsen H, Andersen J, Olsen C, Kragh-Sorensen P. Prevalence of very mild to severe dementia in Denmark. Acta Neurol Scand. 1997;96:82-7.

23. Roelands M, Wostyn P, Dom H, Baro F. The prevalence of dementia in Belgium: a population- 
based door-to-door survey in a rural community. Neuroepidemiology. 1994;13:155-61.

24. Lobo A, Saz P, Marcos G, Dia JL, De-la-Camara C. The prevalence of dementia and depression in the elderly community in a southern european population: the Zaragoza study. Arch Gen Psychiatry. 1995;52:497-506.

25. Pouza SL, Regla JL, Franch V, Pinedo LF. The prevalence of dementia in Girona. Neurologia. 1995;10:189-93.

26. Pi J, Olivé JM, Roca J, Masana L. Prevalence of dementia in a semi-rural population of Catalunya, Spain. Neuroepidemiology. 1996;15:33-41.

27. Lobo A, Launer L, Fratiglioni L, Andersen K, Di Carlo A, Breteler MM, et al. Neurologic diseases in the elderly research Group. Prevalence of dementia and major subtypes in Europe: A collaborative study of population-based cohorts. Neurology. 2000;54 (Suppl 5):S4-S9.

28. Yoshitake T, Kiyohara Y, Kato I, et al. Incidence and risk factors of vascular dementia and Alzheimer's disease in a defined elderly Japanese population. The Hisayama Study. Neurology. 1995;45:1161. 8.

29. Sternberg SA, Wolfson C, Baumgarten M. Undetected dementia in community-dwelling older people: the Canadian Study of Health and Aging. J Am Geriatr Soc. 2000;48:1430-4.

30. Ross GW, Abbott RD, Petrovitch H, Masaki KH, Murdaugh C, Trockman C, et al. Frequency and characteristics of silent dementia among elderly Japanese-American men. The Honolulu-Asia Aging Study. JAMA. 1997;277:800-5.

31. Valcour VG, Masaki KH, Curb JD, Blanchette PL. The detection of dementia in the primary care setting. Arch Intern Med. 2000;160:2964-8.

32. Rocca WA, Bonaiuto S, Lippi A, Luciani P, Turtú F, Cavarzeran F, et al. Prevalence of clinically diagnosed Alzheimer disease and other dementing disorders: a door-to-door survey in Appignano, Macerata province, Italy. Neurology. 1990;40:626631.

33. Ritchie K, Kildea D. Is senile dementia "agerelated" or "ageing related"?- evidence from meta-analysis of dementia prevalence in the oldest old. Lancet. 1995;346:931-4.

34. Herbert LE, Scherr PA, McCann JJ, Beckett LA, Evans DA. Is the risk of developing Alzheimer's disease greater for women than for men? Am J Epidemiology. 2001;153:132-6.

35. Letenneur L, Gilleron V, Commenges D, Helmer C, Orgogozo JM, Dartigues JF. Are sex and educational level independent predictors of dementia and Alzheimer's disease? Incidence data from the PAQUID project. J Neurol Neurosurg Psychiatry. 1999;66:177-83.

36. Letenneur L, Launer L, Andersen K, Dewey ME, Ott A, Copeland JRM, et al . Education and the risk for Alzheimer's disease: sex makes a difference. EURODEM pooled analyses. EURODEM Incidence
Research Group. Am J Epidemiol. 2000;151:106471.

37. Cobb JL, Wolf PA, Au R, White R, D'Agostino RB. The effect of education on the incidence of dementia and Alzheimer's disease in the Framingham Study. Neurology. 1995;45:1707-12.

38. Ngandu NT, Strauss E, Helkala EL, Winblad B, Nissinen A, Tuomilehto J, et al. Education and dementia: What lies behind the association? Neurology. 2007;69:1442-50.

Manuscrito recibido el 28 de noviembre de 2008 y aceptado para publicación el 22 de diciembre de 2008.

Correspondencia:

Dr. Nilton Custodio Capuñay

Unidad de Investigación - Clinica Internacional

Av. Garcilazo de la Vega 1420

Lima 1, Perú

Correo-e: niltoncustodio@neuroconsultas.com 